\title{
Linx
}

Revue des linguistes de l'université Paris X Nanterre

$46 \mid 2002$

Les connecteurs

\section{Mais/pourtant dans la contre-argumentation directe : raisonnement, généricité, et lexique}

Jean-Claude Anscombre

\section{OpenEdition}

Journals

Édition électronique

URL : http://journals.openedition.org/linx/104

DOI : $10.4000 /$ linx. 104

ISSN : 2118-9692

Éditeur

Presses universitaires de Paris Nanterre

Édition imprimée

Date de publication : 1 juin 2002

Pagination : 115-131

ISSN : 0246-8743

\section{Référence électronique}

Jean-Claude Anscombre, « Mais/pourtant dans la contre-argumentation directe : raisonnement,

généricité, et lexique », Linx [En ligne], 46 | 2002, mis en ligne le 25 janvier 2011, consulté le 19 avril 2019. URL : http://journals.openedition.org/linx/104 ; DOI : 10.4000/linx.104 


\title{
Mais/pourtant dans la contre-argumentation directe: raisonnement, généricité, et lexique'
}

\author{
Jean-Claude Anscombre, CNRS et EHESS
}

\section{Introduction : quelques considérations générales}

Ce travail se propose de montrer, par le biais de l'analyse de l'opposition mais/pourtant, que l'on peut avantageusement remplacer la théorie des topoï que nous défendions autrefois ${ }^{2}$ par une théorie des stéréotypes dont les grandes lignes seront précisées plus loin. Je me propose en particulier de remettre en question la validité du critère mais/pourtant comme séparant les topoï intrinsèques des extrinsèques, et de montrer que la notion de déréalisant ${ }^{3}$ peut être intégrée sans problème dans le cadre de cette théorie des stéréotypes.

Je rappellerai pour mémoire que dans la théorie standard des topoï, la langue est fondamentalement de nature argumentative, la relation 'p est un argument pour q' étant considérée comme une primitive du système. Elle fonde les enchaînements argumentatifs, et provient d'un garant $^{4}$, qui, dans le cadre de la théorie standard, prend la forme d'un schéma scalaire et binaire de forme générale $< \pm P, \pm Q>$, ou tout autre notation analogue (appelée dorénavant 'notation topique'). Cette relation argumentative possède deux propriétés essentielles: elle est d'une part dynamique, à savoir qu'un énoncé donné, de par sa nature argumentative, privilégie certains autres énoncés comme de possibles continuations, et en exclut d'autres. Elle opère donc sur le plan syntagmatique. Elle est d'autre part gradable puisqu'un énoncé privilégie de possibles continuations avec plus ou moins de force, ce qui transparaît dans la notation \pm . On ne parle pas pour décrire, mais pour orienter le discours, d'où l'affirmation fréquente que derrière les mots 'il n'y a que d'autres

\footnotetext{
${ }^{1}$ Ce texte est la version française librement adaptée et révisée de Anscombre ; 1998. Je remercie D.Leeman (Université de Paris X-Nanterre) pour la lecture attentive qu'elle a faite de ce texte.

2 Pour un exposé à peu près complet de cette théorie, cf. Anscombre ; 1995c, qui reprend et complète Anscombre ; 1984, 1989. Le concept de topos a été introduit dès Anscombre \& Ducrot ; 1983, mis en œuvre dans Anscombre ; 1984, où sont également étudiés son rôle dans la structuration lexicale et le lien avec les proverbes et expressions sentencieuses (il y a derrière les mots des topoï, en particulier ceux véhiculés par les proverbes et formes sentencieuses).

3 Cf.Ducrot ; 1995.

${ }^{4}$ Notons que le concept de garant n'est pas nouveau, et se rencontre sous des formes diverses chez Perelman et Toulmin. En fait, il apparaît tout à fait explicitement - sous le nom de topos - chez Aristote, dans les Topiques.
} 


\section{Jean-Claude Anscombre}

mots'. La théorie des topoï propose donc - à l'opposé de la plupart des théories actuelles - une sémantique dont le noyau dur n'est pas la référence ${ }^{5}$.

Je voudrais conserver cette vision de la langue - en particulier le clivage entre les niveaux de signification et de référence, tout en en évitant les inconvénients. En effet, et comme je l'ai souligné récemment $^{6}$, le bât blesse dans la représentation choisie par la théorie standard pour illustrer les thèses ci-dessus. Tout d'abord, les $\mathrm{P}$ et Q qui interviennent dans ces schémas sont des 'métaprédicats', i.e. non des prédicats de la langue, mais des concepts ${ }^{7}$ : on est donc loin de l'idée de départ de mots derrière les mots. De plus, ainsi que je l'ai déjà signalé à plusieurs reprises, cette notation renonce d'entrée à la thèse de la signification de base d'un mot comme étant le faisceau des relations (argumentatives) qu'il entretient avec d'autres mots (et n'étant que cela). En effet, la notation topique ci-dessus attribue aux mots des concepts $P, Q, R, \ldots$, indépendants des autres mots, ce qui est contradictoire avec les thèses de départ. Enfin, il s'en faut que la gradabilité inhérente à ces méta-prédicats fournisse les résultats escomptés. Ainsi, si on accepte sans hésitation de représenter un énoncé comme Il faut mettre de l'écran total pour bronzer sans risques sous cette forme standard $< \pm P, \pm Q>$ ('Plus on met d'écran total, moins de risque on prend en bronzant'), on voit mal en revanche comment s'y prendre pour un énoncé pourtant banal comme Il faut le permis pour pouvoir conduire une voiture, qui supposerait un garant du type de 'Plus on a le permis, plus on peut conduire une voiture', dont l'existence est quelque peu sujette à caution. On peut même, à suivre cette théorie au pied de la lettre, aboutir à des prédictions contraires aux faits. Dans Anscombre ; $1996 a^{8}$ j'avais ainsi postulé l'existence d'un schéma topique, un «garant » < $<$ service, \pm payer de retour $>$. Ce qui me permettait d'attacher au mot gratitude un faisceau topique comportant le topos (intrinsèque ${ }^{9}$ ) <+service, +payer de retour $>$. Topos qui me permettait d'expliquer la possibilité de l'enchainement Max n'est pas ingrat : il m'a largement payé de retour pour le service que je lui ai rendu. Or cette façon de procéder contraint pratiquement, pour expliquer l'autre enchaînement Max est un ingrat : je hi ai rendu un grand service, et jattends toujours les remerciements, à attacher à ingratitude le topos <+service, - payer de retour>. Un tel topos présente l'ingrat comme étant celui qui rend d'autant moins qu'on lui a plus donné, ce qui ne correspond pas, il s'en faut, au sens courant du terme.

En fait, un examen attentif des thèses de base révèle aussitôt le défaut de la cuirasse. En particulier, la théorie standard de l'argumentation prend comme acquis que la gradabilité de la relation argumentative (un argument appuie plus ou moins fortement une thèse) est la manifestation de surface de la scalarité des concepts en jeu dans les mots, ce qui revient à faire l'hypothèse d'une scalarité inhérente à tous les concepts attachés aux mots. Or s'il semble raisonnable de voir dans la

\footnotetext{
5 Bien que cette théorie reste référentialiste à sa façon. Elle ne sépare pas totalement le sens et la référence, puisqu'elle admet dans certains cas de dériver les contenus 'informatifs' des contenus 'argumentatifs' (sur ce point, cf. Anscombre \& Ducrot ; 1983, 1986) . Dans la version putnamienne de la théorie des stéréotypes en revanche, les deux fonctions sémantique et référentielle sont totalement séparées. Cf. sur ce point notre article à paraitre « Dénomination, sens et référence dans une théorie des stéréotypes nominaux ».

${ }^{6} \mathrm{Cf}$. Anscombre, 2001a.

${ }^{7}$ De façon tout à fait significative, Ducrot parle de 'propriétés'.

8 Dont l'analogue français se trouve dans Anscombre ; 2001a.

${ }^{9}$ L'hypothèse de mots du lexique comme faisceaux de topoï apparaît dès Anscombre ; 1984, 1989, de façon tout à fait explicite. Dans Anscombre ; 1991 (Actes du Colloque de Cerisy du 22-29 août 1987), est présentée une application de cette thèse à un point mystérieux de grammaire espagnole. Pour les notions de topos intrinsèque et de topos extrinsèque, cf. Anscombre; 1995a, 1995b, 1995 c.
} 
scalarité de certains concepts un moyen de moduler la force argumentative, la thèse inverse n'a aucun caractère d'évidence, ainsi que l'illustrent des cas comme ceux évoqués ci-dessus, et qui ne sont nullement exceptionnels.

Pour conserver les thèses d'un dynamisme non référentialiste de la langue et de la gradabilité du mouvement discursif mais sans prêter le flanc aux critiques exposées plus haut (et ailleurs), j'abandonnerai donc le concept de schéma binaire gradable, au profit d'un autre concept, celui de stéréotype.

\section{Les stéréotypes : quelques notions}

La notion que je vais esquisser brièvement ici reprend l'idée de base de Putnam; 1975, d'un stéréotype attaché aux mots, et celle de Fradin ; 1984, que ce stéréotype est composé de phrases ${ }^{10}$.

Je ferai mienne l'hypothèse de Putnam d'une totale séparation entre la fonction référentielle du langage et la signification intervenant dans le fonctionnement discursif. En d'autres termes, le signifié de base des items lexicaux n'est en aucune façon une description du monde. A certains items lexicaux ${ }^{11}$ est attachée une liste ouverte de phrases, les phrases stéréotypiques, qui constituent le stéréotype de l'item considéré. Par exemple, l'item singe comportera dans son stéréotype la phrase (stéréotypique, donc) Les singes mangent des bananes. D'une façon très générale, si m est un item lexical, son stéréotype sera un ensemble de phrases $g\left(m, n_{1}\right), g\left(m, n_{2}\right), \ldots, g\left(m, n_{i}\right)$, etc., qui relient l'item $m$ en tant que forme à d'autres formes $n_{1}, n_{2}, \ldots, n_{k}$, etc. Et c'est le statut sémantique de ces phrases qui confère une signification à $\mathrm{m}^{12}$. Cette façon de procéder d'une part illustre bien l'hypothèse que derrière les mots il y a d'autres mots, et évite d'autre part le paradoxe inhérent à la notation topique de la théorie standard. Les contenus sémantiques de $m$ et de $n_{1}, n_{2}, \ldots, n_{k}$, etc., ne peuvent être définis indépendamment les uns des autres.

Quelle est la nature des phrases stéréotypiques? Une partie importante d'entre elles sont en fait des phrases génériques. Je rappellerai que, me fondant sur les travaux de Kleiber; 1989, et Anscombre; $1995 \mathrm{c}^{13}$, je distingue trois grands types de phrases génériques, à savoir les analytiques, les typifiantes a priori, les typifiantes locales ${ }^{14}$. Voici un exemple de chacune respectivement :

(1) Les chats sont des mammifères.

(2) Les chats chassent les souris.

(3) Les chats sont affectueux.

Un certain nombre de propriétés les distinguent : j'en ai parlé ailleurs, je n'y reviendrai que brièvement. Une première propriété est la possibilité ou non de prédiquer la propriété (générique)

${ }^{10}$ En fait, Fradin ; 1984, considère qu'un stéréotype est composé d'énoncés, attachés aux mots par un processus semblable à la délocutivité. Ce point ne me paraît pas tenable, comme exposé dans Anscombre ; 2001a, 2001b.

11 Je n'envisage pour l'instant que des items nominaux et verbaux.

12 Ainsi d'ailleurs qu'à $\mathrm{n}_{1}, \mathrm{n}_{2}, \ldots, \mathrm{n}_{\mathrm{k}}$ Admettre Les singes mangent des bananes comme phrase stéréotypique de singe définit la signification de singe, mais aussi de banane, et éventuellement de manger.

${ }^{13}$ Sur la problématique générale de la généricité, cf. Anscombre; 2001, « La nuit, certains chats sont gris, ou la généricité sans syntagme générique «, dans Hommage à M.Galmiche, à paraitre dans Linx.

${ }^{14} \mathrm{La}$ terminologie est quelque peu flottante dans le domaine. Certains rangent également les typifiantes a priori dans les analytiques. A savoir que ce que j'appelle ici analytique serait une analytique nécessairement vraie, la typifiante a priori étant alors une analytique généralement vraie. Quant à la typifiante locale, ce serait une générique synthétique. 


\section{Jean-Claude Anscombre}

d'une entité particulière. En fait, les analytiques et les typifiantes a priori ne permettent pas une telle prédication en tant que marque d'identification d'une entité particulière dans un contexte où la validité de la générique est acceptée :

(3) * Ce chat est un mammifère.

(4) ?? Ce chat chasse les souris ${ }^{15}$.

En revanche, les typifiantes locales - qui représentent en fait les opinions propres au locuteur - admettent la prédication particularisante :

(5) Ce chat est affectueux.

A bien y regarder, cette propriété n'est autre que le principe bien connu qu'on ne saurait caractériser une entité particulière au moyen de caractéristiques constitutives générales ${ }^{16}$ de la classe d'appartenance. Une autre caractéristique différentielle bien connue est la possibilité de donner lieu à des raisonnements syllogistiques bien formés ${ }^{17}$. Ainsi, on trouvera sans doute impeccable le raisonnement suivant :

(6) Les chats sont des mammifères Cet animal est un chat

(Donc) cet animal est un mammifère.

dont la prémisse est une phrase générique analytique. Considérons maintenant la phrase cette fois typifiante a priori Les singes mangent des bananes. On remarque immédiatement que si l'on tente le même raisonnement qu'en (6), avec par exemple Cheetah est un singe comme mineure, la conclusion (Donc) Cheetah mange des bananes paraît quelque peu forcée. On attendrait en fait des choses comme Cheetah mange (vraisemblablement + probablement) des bananes, i.e. une conclusion non pas certaine mais probable. On vérifiera qu'il en est de même pour les typifiantes locales. C'est que les analytiques présentent une propriété comme nécessairement vraie, alors que les typifiantes ne la présentent que comme généralement vraie. Elles admettent donc d'éventuelles exceptions, sans pour autant perdre leur généricité. D'où le contraste :

(7) * Les chats sont des mammifères, sauf le mien, Minet. Les chats chassent les souris, sauf le mien, Minet, qui est végétarien. Les chats sont affectueux, sauf les chats siamois.

\footnotetext{
${ }^{15}$ La bizarrerie de (3) est patente, mais je m'arrêterai un instant sur (4). On remarquera que je n'ai pas dit que (4) était strictement impossible, mais qu'il ne peut apparaitre dans des contextes comme :

(4a) - jai acheté un chat.

- Et comment il est, ce chat?

- Il chasse les souris.

La dernière réplique est bizarre, sauf à la comprendre comme 'Il passe son temps à chasser les souris', pour des raisons qui apparaitront plus loin. En revanche, on peut rappeler que cette caractéristique est inhérente à la classe considérée :

(4b) Comme tous les chats, ce chat chasse les souris.

D'une façon analogue, la réponse Elle a quatre roues à la question Comment est ta nouvelle voiture? ne sera jamais comprise comme destinée à caractériser la voiture.

16 On peut se demander à ce propos s'il n'y a pas là l'origine de la notion de trait distinctif à la Saussure. Seuls comptent pour le sens les traits qui justement ne font pas partie des caractéristiques constitutives générales de la classe.

17 Cf. Kleiber ; 1988.
} 
Les phrases analytiques sont en fait la base du discours scientifique, et s'appuient sur des lois purement logiques. Les phrases typifiantes, au contraire, relèvent d'un autre registre, celui du discours commun. Elles présentent des conclusions comme probables, comme acquises in absentia. Les deux types de discours s'opposent par le type de garant utilisé. Le discours logique utilise des phrases génériques analytiques, et il n'est pas argumentatif. En revanche, le discours dont le déroulement repose sur des phrases typifiantes est un discours argumentatif, mais non logique, puisque le garant peut présenter des exceptions. On peut donc dire que l'argumentativité provient de la nature typifiante du garant sur lequel repose l'enchaînement discursif, et que cette nature typifiante provient à son tour de l'existence de possibles exceptions. Je m'appuierai sur cette remarque pour définir une notion de force argumentative qui ne soit pas nécessairement liée à la gradabilité des prédicats (ou des méta-prédicats qui les représentent). A cet effet, supposons qu'un mot m comporte dans son signifié (entre autres choses) la phrase générique typifiante $\mathrm{g}(\mathrm{m}, \mathrm{n})$, qui met en relation $\mathrm{m}$ avec $n$. Un exemple typique d'une telle situation est le cas des phrases génériques de type Les $m$ possèdent la propriété $n$. Nous dirons alors que: a) L'usage de m argumente en faveur de la propriété $n$ avec une certaine force argumentative $f$; $)$ Cette force argumentative $f$ est fonction du nombre plus ou moins important d'exceptions au principe général exprimé par $\mathrm{g}(\mathrm{m}, \mathrm{n})$ envisagées par le locuteur quand il utilise $m$ en faveur de $n$. Considérons par exemple les enchaînements suivants :

(8) Cheetah est un singe : elle aime peut-être les bananes.

(9) Cheetab est un singe : elle doit aimer les bananes.

(10) Cheetab est un singe : elle aime certainement les bananes.

dans un contexte où est admise la validité de la phrase générique :

(11) $\mathrm{g}($ singe, banane $)=$ Les singes aiment les bananes .

On remarque que ces enchainements ne se différencient que par la présence d'une modalité relative à la force argumentative appliquée. Selon les connaissances du locuteur quant aux habitudes alimentaires des singes, et ses doutes en la matière, il utilisera (8), (9), ou (10) en fonction des exceptions qu'il sera disposé à admettre. Nous évitons ainsi d'avoir à attribuer à singe une forme topique <+singe, +bananes>, dont le ridicule est évident, et la pertinence un tant soit peu discutable. Mais il y a plus. Nous pouvons maintenant expliciter le lien entre la gradabilité d'un prédicat linguistique et la gradabilité de la force argumentative liée à son usage. Le locuteur de Je suis un peu fatigué renvoie, au travers de l'occurrence de fatigué, à une phrase générique typifiante, par exemple Les gens fatigués ne sortent pas le soir. Il utilise le faible degré exprimé par un peu pour indiquer qu'il envisage quelques exceptions à ce principe. C'est ce qui fait de l'énoncé ci-dessus un argument faible pour Je ne pense pas sortir ce soir. On obtiendrait un discours beaucoup plus convaincant à l'aide de Je suis très fatigué ou encore Je suis épuisé.

Nous allons maintenant utiliser cette série de remarques dans le cas particulier de l'opposition mais/pourtant.

\section{Vers une analyse stéréotypique de l'opposition mais/pourtant}

Les lignes qui suivent seront consacrées à l'analyse de cette opposition dans le cadre de la théorie des stéréotypes. Il s'agit de caractériser les propriétés sémantiques des structures de type $p$ mais $q$ et $p$, (et) pourtant $q$, dans le but d'établir d'éventuelles différences entre ces deux conjonctions. Pour des raisons de longueur, je ne m'intéresserai ici qu'aux occurrences complètes de ces structures, i.e. je passerai sous silence les cas où manquent soit l'antécédent, soit le conséquent, 


\section{Jean-Claude Anscombre}

soit les deux. En d'autres termes, je fais l'hypothèse que l'analyse des structures complètes est pertinente pour la description des structures incomplètes, et que, en particulier, elle permet éventuellement la reconstruction des membres manquants.

Je commencerai par reprendre, en l'aménageant, une distinction déjà présente dans Anscombre-Ducrot ; 1978, 1979, 1983, Anscombre ; 1983 :

Définition : soit $p$ Conec $q$ une séquence discursive où $p$ est l'antécédent, et $q$ le conséquent ${ }^{18}$, et Conec le connecteur qui les met en relation. Je dirai que cette séquence présente une contreargumentation indirecte s'il existe une conclusion $r$, distincte de $p$, conclusion pour laquelle $q$ est argument, $p$ étant argument cette fois pour $\sim r$. La contre-argumentation est directe si $p$ est argument pour $\sim q$.

Voici quelques exemples de contre-argumentation :

(12) «....Ne pas se laisser dépasser par les sentiments, aussi admirables, aussi légitimes soientils...» (Daniel Rops).

(13) «...Quoique souffrant, je suis sorti... » (Littré).

(14) «...Bien qu'ayant vécu chez eux, je connais mal ces ennemis du genre humain...» (A.France)

(15) «...Vous n'êtes pas forcé de me répondre, quoique cela me ferait le plus grand plaisir... » (G.Sand).

(16) «...Je sais que les hommes ne sont que des hommes, quand bien même ils sont très grands... »(G.Duhamel).

(17) «...Ils fuyaient le voisinage des habitations, et redoutaient pourtant la solitude...» (Flaubert).

(18) «...Dans l'espace élargi que le regard parcourt, l'œuvre est certes un objet privilégié, mais elle n'est pas le seul objet qui s'impose à la vue... » (Starobinski).

(19) «... Joanny s'était senti tout fier d'exciter la curiosité de la jeune fille. "Elle va m'étudier, pensait-il". Il aurait voulu rendre sa conduite, exprès, singulière ; mais il avait trop peur d'être ridicule... » (Larbaud).

(12) est une contre-argumentation directe : éprouver des sentiments admirables et légitimes induit à se laisser dépasser [par ces sentiments], ce qui s'oppose directement à ne pas se laisser dépasser. De même dans (13), être souffrant convoque la phrase générique du type Quand on est souffrant, on ne sort pas, d'où l'on tire immédiatement une opposition directe avec je suis sorti. (14) enfin est également une opposition directe, par le biais de Quand on vit chez quelqu'un, on le connât bien. (15) exhibe en revanche une opposition cette fois indirecte: Vous n'êtes pas forcé de répondre est argument pour Ne répondez pas, alors que cela me ferait le plus grand plaisir est cette fois argument pour Répondez. (16) est une opposition directe, au travers de Les grands hommes sont plus que des hommes, de même que (17) : fuir le voisinage des habitations est un argument pour redouter la compagnie des hommes, qui s'oppose donc directement à redouter la solitude. (18) et (19) sont intéressants parce que le premier recèle une opposition directe, et le second une opposition cette fois indirecte. En effet, être un objet privilégié est argument pour être un objet unique, qui s'oppose donc, en bref, à ne pas être le seul objet. Dans (19), il aurait voulu rendre sa conduite

${ }^{18}$ Ce ne sont donc pas nécessairement les segments matériels situés avant ou après Conec. 
singulière est argument pour il va exciter la curiosité de la jeune fille, et il avait peur d'être ridicule est argument pour il ne va pas exciter la curiosité de la jeune fille.

Or si l'opposition argumentation directe/argumentation indirecte paraît intuitivement assez claire, il faut bien admettre que pour l'instant, aucun critère un peu précis ne nous permet de faire le départ entre les deux types d'argumentation. Pour jeter quelque lumière sur le sujet, j'examinerai maintenant quelques propriétés distributionnelles et sémantiques des structures contreargumentatives, en me limitant aux connecteurs mais et pourtant.

Une première question concerne la définition même de la contre-argumentation dans une séquence $p$ Conec $q$. Pourquoi en effet choisir la condition 'p est argument pour q' plutôt que 'q est argument pour $\sim p^{\prime}$, ou encore '(p est argument pour $\sim q$ ) ou (q est argument pour $\sim$ )' ? Il n'y a en effet aucune raison apparente de préférer l'une à l'autre. Pour justifier le choix fait, je commencerai par introduire deux lois rhétoriques banales, à savoir : $\left(\mathrm{L}_{1}\right) \mathrm{Si} \mathrm{x}$ est argument pour $\mathrm{y}$, alors $\sim \mathrm{x}$ est argument pour $\sim y ;\left(\mathrm{L}_{2}\right) \mathrm{Si} x$ est argument pour $\mathrm{y}$, alors y est argument pour $\mathrm{x}$. Illustrons ces deux lois sur la pertinence desquelles nous reviendrons plus avant. Supposons par exemple que je considère que savoir que Victor Hugo est l'auteur des Misérables est faire preuve de culture, alors je serai probablement enclin à penser qu'ignorer cette parenté est un indice d'inculture, ce qui est faire jouer $\left(\mathrm{L}_{1}\right)$. Considérons maintenant la phrase générique Les singes mangent des bananes. Il s'agit, nous l'avons vu, d'une phrase typifiante (a priori), et comme telle, elle admet donc de possibles exceptions. Par conséquent, le mouvement discursif qui, partant de cette prémisse générique et de $S$ est un singe, aboutit à $S$ mange des bananes est argumentatif au sens donné plus haut à ce terme. Supposons maintenant, dans le cadre de la même phrase générique, que $S$ soit un animal inconnu, mais dont on sait qu'il est grand consommateur de bananes. Nous aurions alors tendance à tirer la conclusion $S$ mange des bananes, ce doit être un singe, i.e. à appliquer $\left(\mathrm{L}_{2}\right)$. Si donc 'p est argument pour $\sim \mathrm{q}$ ', alors ' $\sim$ p est argument pour $\sim \sim q=q^{19}$ d'après $\left(\mathrm{L}_{1}\right)$. D'où l'on tire alors, selon $\left(\mathrm{L}_{2}\right)$, que 'q est argument pour $\sim$ p'. Par conséquent, les différentes possibilités que nous avons mentionnées plus haut se déduisent les unes des autres par le jeu des lois rhétoriques les plus usuelles. Pourquoi donc privilégier la forme 'p est argument pour q' ? Une première remarque sera que si des lois comme $\left(\mathrm{L}_{1}\right)$ et $\left(\mathrm{L}_{2}\right)$ conservent la relation argumentative, elles n'en sont pas pour autant symétriques, en particulier au niveau des conclusions tirables, car la force argumentative n'est pas nécessairement conservée. Ainsi, si p est un argument fort pour q, alors généralement, p sera un argument faible pour $\sim$ q. Ce qui revient à dire que si de p on peut tirer la conclusion $q$, il peut se faire que de $\sim$ on ne puisse tirer la conclusion $\sim q$. Ainsi, la grande majorité des mathématiciens accepteraient de dire Max a démontré le théorème de Fermat : c'est un grand mathématicien, qui rechigneraient à dire Max n'a pas démontré le théorème de Fermat: ce n'est pas un grand mathématicien - la liste des grands mathématiciens qui se sont attaqués sans succès à ce théorème étant fort longue. On comprend ainsi que le choix opéré ne soit pas indifférent au niveau de la conclusion. Le choix que nous avons fait correspond généralement à la distribution de surface, même si un tel argument n'est pas décisif. Ainsi, dans un exemple comme Je ne suis pas content : Lia est venue bier, mais elle était avec sa mère, le segment Je ne suis pas content est présenté comme la conclusion de Lia était avec sa mère - et non l'inverse, Lia est venue bier étant argument pour je suis content, sans entrer dans les détails. L'origine de cette dissymétrie transparaît à la lueur de la définition suivante :

Définition : si p et q sont deux phrases, je dirai que p est argument pour q dans un enchainement discursif $\mathrm{p}+\mathrm{q}$, selon le garant $\mathrm{g}(\mathrm{p}, \mathrm{q})$, si :

\footnotetext{
${ }^{19} \mathrm{Si}$ on admet la loi de double négation, qui est en fait valable en langue sous certaines conditions.
} 
Jean-Claude Anscombre

$\mathrm{g}(\mathrm{p}, \mathrm{q})$ est une phrase générique reliant $\mathrm{p}$ à $\mathrm{q}^{20}$.

La conjonction de $\mathrm{g}(\mathrm{p}, \mathrm{q})$ et de p permet éventuellement de tirer la conclusion $\mathrm{q}$.

Notons que le fait de pouvoir tirer une conclusion n'implique pas que cette conclusion soit convaincante, cette caractéristique dépendant de la force argumentative. Avec une telle définition, qui inscrit l'argumentation dans le cadre plus général de la théorie des stéréotypes, les notions d'argumentation et de phrase générique typifiante sont intimement liées, et j'utiliserai l'une ou l'autre des deux formes, selon la commodité d'expression. Ainsi, l'enchaînement Cet animal est un singe, il doit manger des bananes peut être interprété comme un enchaînement argumentatif via $\mathrm{g}(\mathrm{p}, \mathrm{q})=$ Les singes mangent des bananes. Remarquons que, malgré l'absence de connecteur, il y a une interprétation préférentielle de l'enchaînement, qui est compris comme un donc plutôt que comme un en effet ou un puisque ${ }^{21}$. Soit maintenant :

(20) Max est un ingrat : je lui ai rendu un grand service, et il ne m'a même pas remercié.

Par commodité, j'utiliserai les notations suivantes : $\mathrm{p}_{1}=$ j'ai rendu un grand service à Max; $\mathrm{p}_{2}=$ Max ne m'a même pas remercié; $\mathrm{q}_{1}=$ Max doit montrer sa reconnaissance; $\mathrm{q}_{2}=$ Max est un ingrat. Pour les garants, je proposerai les phrases génériques : $\mathrm{g}_{1}=$ quand on vous rend service, on doit montrer sa reconnaissance; $\mathrm{g}_{2}=$ remercier est la manifestation minimum de la reconnaissance $; \mathrm{g}_{3}=$ qui manifeste de la reconnaissance n'est pas un ingrat. Selon le garant $\mathrm{g}_{1}$, $\mathrm{p}_{1}$ est argument pour la conclusion $\mathrm{q}_{1}$. De $\mathrm{g}_{2}$, et moyennant l'intervention de la loi $\left(\mathrm{L}_{1}\right)$, on tire Qui ne remercie pas ne manifeste aucune reconnaissance. De $\mathrm{g}_{3}$, et toujours moyennant $\left(\mathrm{L}_{1}\right)$, on tire Qui ne manifeste aucune reconnaissance est un ingrat. En combinant $\mathrm{g}_{2}$ et $\mathrm{g}_{3}$ sous leur forme négative, on aboutit à $g_{4}=$ Qui ne remercie pas est un ingrat. De ce dernier, combiné avec $\mathrm{p}_{2}$, on tire Max est un ingrat, i.e. $\mathrm{q}_{2}$. Mais il y a plus : nous avons vu que $\mathrm{p}_{1}$ est argument pour $\mathrm{q}_{1}=$ Max doit montrer sa reconnaissance. Par ailleurs, de $\mathrm{p}_{2}$, et moyennant la version négative de $\mathrm{g}_{2}$, on tire $\mathrm{q}_{2}^{\prime}=$ Max n'a montré aucune reconnaissance, conclusion opposée à $\mathrm{q}_{2}$. Nous voyons ainsi que $\mathrm{p}_{1}$ et $\mathrm{p}_{2}$ sont d'orientations argumentatives opposées, d'où la présence du et parfois appelé et d'opposition, reliant $\mathrm{p}_{1}$ et $\mathrm{p}_{2}$, et destiné précisément à marquer l'opposition de deux arguments, rôle dans lequel il est fréquemment combiné à pourtant. On vérifiera d'ailleurs que l'insertion d'un pourtant après le et dans (20) convient parfaitement pour le sens visé. Au vu de tels exemples, je ferai l'hypothèse suivante :

Thèse :

a) Dans un enchaînement de type $p$, mais $q$, mais peut introduire une contre-argumentation directe ou indirecte.

b) Dans un enchaînement de type $p$, (et) pourtant $q$, (et) pourtant introduit une contreargumentation directe.

c) Dans le cas d'une contre-argumentation directe, tant mais que pourtant s'appuient sur un garant $\mathrm{g}(p, \sim q)$.

Les points a) et b) ne sont absolument pas nouveaux, et je me contenterai de les illustrer sur les exemples suivants :

\footnotetext{
20 Je simplifie à dessein. Il faudrait dire qu'il existe une phrase générique $\mathrm{g}(\mathrm{P}, \mathrm{Q})$, reliant $\mathrm{P}$ et $\mathrm{Q}$, p et q pouvant être considérés comme des particulières de $\mathrm{P}$ et de $\mathrm{Q}$ respectivement. Par ailleurs, $\mathrm{p}$ et $\mathrm{q}$ peuvent ne pas être des phrases, mais des segments, par exemple des prédicats comme 'être un singe'.

21 La présence d'une anaphore n'est sans doute pas complètement étrangère à cette assymétrie.
} 
Mais/pourtant dans la contre-argumentation directe : raisonnement, généricité et lexique

(21) Je ne prendrai pas de dessert : j'adore le sucré, mais ça fait grossir.

(22) Mon chat n'est en rien une exception : il est affectueux, mais tous les chats le sont.

(23) Les autruches sont des oiseaux, mais elles ne volent pas.

(24) Max fume comme un pompier, mais il ne tousse absolument pas.

Alors que (21) et (22) sont clairement des contre-argumentations indirectes, (23) et (24) peuvent facilement s'interpréter comme des contre-argumentations cette fois directes. Elles s'appuient sur les phrases génériques Les oiseaux volent, et Les fumeurs toussent respectivement. On remarque alors que pourtant est clairement substituable à mais dans (23) et (24), manipulation quasiment impossible dans le cas de (21) et (22) :

(25) * Je ne prendrai pas de dessert : j'adore le sucré, pourtant ça fait grossir.

(26) * Mon chat n'est en rien une exception: il est affectueux, pourtant tous les chats le sont.

(27) Les autruches sont des oiseaux, pourtant elles ne volent pas.

(28) Max fume comme un pompier, pourtant il ne tousse absolument pas.

Pour ce qui est du point c), j'en démontrerai la pertinence par l'impossibilité d'enchaînements reposant sur des garants qui ne correspondraient à aucun savoir partagé. Commençons sur les exemples suivants, qui font intervenir pourtant :

(29) * Lia est mariée, et pourtant, elle veut des enfants.

(30) ?? Mon chat aime la viande, et pourtant, il n'aime pas les légumes.

(31) ?? Max est d'une famille très religieuse, et pourtant, il s'est marié à l'église.

Si de tels exemples sont, dans notre culture, plutôt discutables, c'est qu'ils reposeraient, s'ils étaient possibles, sur des garants tout aussi discutables, à savoir Les gens se marient pour ne pas avoir d'enfants, Les animaux qui aiment la viande aiment aussi les légumes, et Les gens qui appartiennent à une famille très religieuse ne se marient pas à l'église. Notons que si nous rétablissons les configurations correspondant aux garants usuels, on obtient alors des enchainements tout à fait banals :

(32) Lia est mariée, et pourtant, elle ne veut pas d'enfants.

(33) Mon chat aime la viande, et pourtant, il aime les légumes.

(34) Max est d'une famille très religieuse, et pourtant, il ne s'est pas marié à l'église.

Notons enfin que les exemples (29) à (31) sont possibles si on substitue mais à pourtant, et qu'on les interprète comme des contre-argumentations cette fois indirectes :

(35) [Le mariage ne dégoûte pas forcément les gens de la vie de famille :] Lia est mariée, mais elle veut (quand même) des enfants.

(36) [Mon chat est difficile à nourrir :] il aime la viande, mais il n'aime pas les légumes.

(37) [Les enfants de familles religieuses ne sont pas toujours anti-religion:] Max est d'une famille très religieuse, mais il s'est (quand même) marié à l'église 22 .

22 Beaucoup de ces exemples sont meilleurs avec mais quand même qu'avec mais seul. 
Jean-Claude Anscombre

Considérons finalement les exemples :

(38) Cest bizarre :Titi est un oiseau, mais il ne vole pas.

(39) Cest bizarre : Titi est un oiseau, (et) pourtant, il ne vole pas.

(40) ?? C'est bizarre : Titi ne vole pas, mais c'est un oiseau.

(41) C'est bizarre :Titi ne vole pas, (et) pourtant, c'est un oiseau.

(42) Cest bizarre :Titi est un oiseau, mais pourtant, il ne vole pas.

(43) ?? C'est bizarre : Titi ne vole pas, mais pourtant c'est un oiseau.

On voit ce qui apparaît à travers ces exemples : d'une part, la substitution de mais à pourtant n'est pas toujours possible, comme le montre l'opposition entre (41) et (40). La possibilité de Titi doit avoir des plumes: il ne vole pas, mais c'est un oiseau, nous montre que dans (40), la substitution de mais à pourtant ne permet pas (toujours) de conserver l'opposition directe, puique (40) redevient possible avec une conclusion imposant une opposition indirecte. On remarque alors qu'avec cette nouvelle conclusion, c'est (41) qui devient bizarre : ?? Titi doit avoir des plumes : il ne vole pas, (et) pourtant, c'est un oiseau. Si nous comparons maintenant (39) et (41), on s'aperçoit qu'ils ne diffèrent que par les places de $p=$ Titi est un oiseau, et $q=$ Titi ne vole pas, qui ont été interchangées. Une telle opération est en revanche impossible avec mais ((38) versus (40)), même renforcé par pourtant ((42) versus (43)). On voit ainsi apparaître un argument supplémentaire appuyant notre choix : s'il peut se faire qu'il y ait une certaine symétrie entre ' $p$ est argument pour $q$ ' et ' $q$ est argument pour $\sim p$ ', il n'en est pas toujours ainsi. Et ce que montrent les exemples cidessus, c'est qu'est toujours possible l'option ' $p$ est argument pour $\sim q$ ', laquelle correspond d'ailleurs à l'ordre d'apparition des caractéristiques dans la phrase générique de base Les oiseaux volent.

Dernière observation pour clore ce paragraphe : dans le cas de mais, outre une intuition qui n'est pas toujours un bon guide, et l'examen d'une conclusion globale qui n'est pas toujours facile à déterminer, un critère assez fiable de contre-argumentation directe est la possibilité de combiner ce mais avec un pourtant. Ainsi, Titi est un oiseau, mais pourtant il ne vole pas, est possible avec la conclusion globale C'est bizarre - (38) est donc une contre-argumentation directe. Et impossible avec Titi ne s'échappera pas - qui sert alors une contre-argumentation indirecte.

\section{Mais/pourtant, exceptions, et topoï intrinsèques}

\subsection{Exceptions ordinaires et exceptions extraordinaires}

Jusqu'à présent, nous avons essentiellement examiné les ressemblances entre mais et pourtant dans la contre-argumentation directe. Nous allons maintenant nous consacrer à l'analyse d'une différence à mes yeux essentielle entre ces deux connecteurs, toujours dans le cas de la contreargumentation directe, et en usage uniquement monologal23. Je voudrais soutenir l'hypothèse suivante :

23 Pour la caractérisation des usages monologaux et monologiques des connecteurs de contre-argumentation, cf. Portolés; 1995. 
Thèse :

a) Dans une structure $p$, mais $q$, mais présente q comme contraire au conséquent que l'on peut inférer de p et du garant $\mathrm{g}(\mathrm{p}, \sim \mathrm{q})$. Une telle exception au principe exprimé dans $\mathrm{g}(\mathrm{p}, \sim \mathrm{q})$ n'en infirme pas la validité générale.

b) Dans une structure $p$, (et) pourtant $q$, (et) pourtant présente q comme contraire au conséquent que l'on peut inférer de $\mathrm{p}$ et du garant $\mathrm{g}(\mathrm{p}, \sim \mathrm{q})$. La coexistence de $\mathrm{p}$ et de $\mathrm{q}$ apparait comme une exception qui remet en question le principe exprimé dans g $(p, \sim q)$, et en infirme donc la validité générale.

En résumé, mais présente une exception qui reste compatible avec la règle, qui ne fait pas sortir du cadre défini par le garant générique convoqué. (Et) pourtant, en revanche, interroge la validité de la règle convoquée, et situe le débat hors de ce cadre. De façon quelque peu lapidaire, on peut dire que l'exception que présente mais en quelque sorte confirme la règle, il s'agit d'une exception ordinaire. Pourtant présente en revanche une exception qui infirme la règle, et que nous appellerons exception extraordinaire.

Considérons les exemples :

(44) Max et Lia sont mariés, mais ils n'ont pas d'enfants.

(45) Max et Lia sont mariés, et pourtant ils n'ont pas d'enfants.

D'une telle paire minimale, il serait tentant de déduire que le contraste entre mais et pourtant est en fait de nature purement stylistique. En fait, chaque connecteur suppose un contexte d'utilisation bien spécifique. Supposons par exemple une enquête cherchant à établir le nombre moyen d'enfants par famille. A la question Max et Lia sont mariés, combien ont-ils d'enfants?, c'est clairement (44) qui conviendrait, et non (45). En effet, la question s'inscrit - par le biais d'un présupposé - dans le cadre du garant Les couples mariés ont des enfants. Par rapport à ce garant, on ne peut donc évoquer que des exceptions ordinaires, les seules à le conserver : c'est donc (44) qui convient, et non (45). Supposons maintenant que le maire d'une station thermale, à seule fin de vanter les mérites de son eau, se vante de ce que dans son village, Les couples mariés ont des enfants. Un concurrent jaloux désireux de montrer que l'eau en question ne vaut rien, utiliserait préférentiellement (45). En effet, (45) présente selon nous une exception extraordinaire, qui remet donc en question le garant ci-dessus. Une dernière remarque pour conclure ce paragraphe : alors qu'intervertir $p$ et q change peu de choses à l'interprétation de (45), la même opération appliquée à (44) produit un résultat fondamentalement différent :

(46) Max et Lia n'ont pas d'enfants, mais ils sont mariés.

(46) étant régulièrement interprété comme une contre-argumentation indirecte. Par exemple, pour une conclusion Max et Lia ont fini par faire comme tout le monde, à laquelle s'oppose Max et Lia n'ont pas d'enfants, et à laquelle conduit Ils sont mariés.

Je voudrais revenir pour terminer sur les exemples (38) à (43), qui posent plusieurs problèmes. Un premier problème est que, alors que p et q peuvent permuter avec pourtant, ils ne le peuvent avec mais. Ce que l'on a parfois exprimé en disant que pourtant était symétrique, et que mais ne l'était pas. C'est qu'en fait les deux mécanismes sont fondamentalement différents. Le rôle de pourtant est d'invalider un garant en affirmant son incompatibilité avec un certain phénomène, une exception extraordinaire. Dans la mesure donc où il s'agit de constater une divergence irrémédiable, l'ordre d'apparition des éléments est de peu d'importance. C'est d'ailleurs déjà ce qui se passe avec le et d'opposition, qui lui aussi admet la permutation :Tu exagères : (tu me traites comme moins que 
Jean-Claude Anscombre

rien, et tu me demandes de t'aider + tu me demandes de t'aider, et tu me traites comme moins que rien). Mais fonctionne de façon radicalement différente : son rôle est en effet de préserver un garant, en présentant un phénomène comme n'étant rien de plus qu'une exception ordinaire. Or nous avons vu que de ce garant, on tire ' $p$ est argument pour $\sim q$ ', mais non ' $q$ est argument pour $\sim p$ '. On ne peut permuter. Ceci dit, comment se fait-il qu'on ait le phénomène signalé en :

(40)?? C'est bizarre : Titi ne vole pas, mais c'est un oiseau.

En effet, à partir du garant Les oiseaux volent, rien n'empêche de déduire Ce qui ne vole pas n'est pas un oiseau, par application successive des lois $\left(\mathrm{L}_{1}\right)$ et $\left(\mathrm{L}_{2}\right)$ vues plus haut. On aurait alors bel et bien une contre-argumentation directe ${ }^{24}$. La raison en est en fait à chercher dans un principe très général, à savoir que ce qu'on dérive d'un garant par les lois habituelles n'est pas nécessairement un garant. En particulier, une entité est caractérisée par les propriétés qu'elle a, et non celles qu'elle n'a pas. C'est pourquoi la fameuse définition bomme = bipède sans plumes fait habituellement sourire, et c'est une raison analogue qui est à l'origine du phénomène signalé dans (40).

Autre problème : nous avons dit que mais présentait q comme une exception ordinaire, i.e. confirmant la règle $\mathrm{g}(\mathrm{p}, \sim \mathrm{q})$, alors que pourtant voyait dans $\mathrm{q}$ une exception extra-ordinaire, infirmant cette même règle. Dans ces conditions, mais et pourtant sont par nature incompatibles, et ne devraient pas pouvoir être combinés. Or on a sans problème :

(42) C'est bizarre : Titi est un oiseau, mais pourtant, il ne vole pas.

Une première remarque est que si (42) est possible, il est aussi possible sans pourtant, et comme contre-argumentation directe: c'est l'énoncé déjà vu (38). Une seconde remarque sera que sans pourtant, mais peut donner lieu à une argumentation indirecte, par exemple : Titi ne risque pas de s'échapper de sa cage: c'est un oiseau, mais il ne vole pas. On voit alors quelle sera notre solution : elle consistera à dire que dans (42), mais introduit en fait une contre-argumentation indirecte, sur laquelle renchérit pourtant par une contre-argumentation directe. Mais dit que Titi est un oiseau est un argument pour 'On peut appliquer le garant $\mathrm{g}=$ les oiseaux volent', et que Titi ne vole pas représente une exception ordinaire à ce principe général, qui fait qu'on ne peut appliquer ce garant. Ce qu'ajoute alors pourtant, c'est que si on ne peut pas appliquer le garant g, c'est parce que Titi ne vole pas montre que $\mathrm{g}$ n'est pas valide. Mais agit en quelque sorte au niveau métalinguistique, et concerne l'applicabilité ou non d'un garant au cas particulier envisagé. Pourtant intervient alors pour dire que la question ne se pose pas, car le cas particulier en jeu montre que le garant envisagé n'est pas valide.

\subsection{Topoï intrinsèques et opérateurs déréalisants}

Comme je l'ai dit dans l'introduction, un problème important est celui de la pertinence des notions de topos intrinsèque et d'opérateur déréalisant. Je commencerai par rappeler brièvement les définitions et propriétés principales concernant ces deux notions. Etant donné un topos reliant les termes $m$ et $n$ - respectivement initial et final - d'un topos, le topos sera dit intrinsèque si l'enchaînement $m$, mais $\sim n$ est acceptable, et si l'enchaînement cette fois $m$, mais $n$ ne l'est pas. Si les deux enchaînements sont possibles, le topos est alors dit extrinsèque. Si de plus l'enchaînement

24 On notera que (40) redevient possible en contre-argumentation indirecte : Titi fera l'affaire dans ce film: il ne vole pas, mais c'est un oiseau. 
$m$, (et ) pourtant $\sim n$ est acceptable, alors le topos ${ }^{25}$ est extrinsèque. Illustrons cette opposition sur les exemples :

(46) Il y a un problème, mais il est facile à résoudre.

(47) ?? Il y a un problème, mais il est difficile à résoudre.

(48) ?? Il y a un problème, pourtant il est facile à résoudre.

(49) Il y a un problème, mais le chef s'obstine à ne rien faire.

(50) Il y a un problème, mais le chef s'en occupe.

(51) Il y a un problème, (et) pourtant, le chef s'obstine à ne rien faire.

Selon (46), (47) et (48), c'est un topos intrinsèque lié à problème qu'un problème soit difficile à résoudre, et c'est un topos cette fois extrinsèque que s'il y a un problème, c'est au chef de s'en occuper, bien qu'il s'agisse là d'un lieu commun de la vie quotidienne. Passons maintenant au concept de déréalisant. Nous dirons qu'une unité lexicale $\mathrm{Y}$ est déréalisante pour une expression $\mathrm{X}$ si :

a) La combinaison $\mathrm{X}+\mathrm{Y}$ n'est pas contradictoire.

b) La combinaison $\mathrm{X}+\mathrm{Y}$ a une orientation argumentative inverse de ou une force argumentative inférieure à celle de $\mathrm{X}$ seule.

Examinons par exemple :

(52) Les travaux de la maison avancent, et même rapidement.

(53) ? Les travaux de la maison avancent, et même lentement.

(54)? Les travaux de la maison avancent, mais rapidement.

(55) Les travaux de la maison avancent, mais lentement.

On voit ainsi que lentement est déréalisant pour avancer, avec une orientation inverse qui explique (53) et (55). En revanche, rapidement est réalisant pour avancer : il entraine une orientation argumentative de même sens et supérieure.

Des deux définitions qui précèdent, il ressort immédiatement que les deux notions de topos intrinsèque et d'expression déréalisante sont corrélées, du moins lorsque, comme ici, un adverbe $\mathrm{A}$ modifie un prédicat $P$. Les trois caractéristiques suivantes sont ainsi équivalentes :

(i) $(+\mathrm{P},+\mathrm{A})$ est un topos intrinsèque de $\mathrm{P}$.

(ii) $\mathrm{A}$ est réalisant pour $\mathrm{P}($ et $\sim \mathrm{A}$ déréalisant de $\mathrm{P})$.

(iii) Les enchaînements :

a) $P$, et même $(P+A) ; P$, mais $(P+\sim A)$ sont possibles.

Et les enchaînements :

b) $\quad$, et même $(P+\sim \mathrm{A}) ; P$, mais $(P+A)$ sont impossibles.

25 Et plus généralement le garant, ce qui est en jeu ici est l'opposition intrinsèque/extrinsèque. 
Jean-Claude Anscombre

Equivalence qu'on peut vérifier sur les enchaînements suivants :

(56) Les travaux de la maison avancent, (mais + ??pourtant) ils avancent lentement.

(57) Il y a un problème, (mais + ??pourtant) il est facile à résoudre.

(58) Max boit, (mais + *pourtant) il boit avec modération.

Dans de tels exemples, pourtant ne peut remplacer mais, ce qui semble conforme à ce que nous avons dit des topoï intrinsèques. Or il y a des cas de topoï intrinsèques - et nullement exceptionnels, où cette substitution est possible, ainsi :

(59) Max a cherché une solution, et pourtant, il ne l'a pas trouvée.

(60) Lia a frappé à la porte, et pourtant, on ne lui a pas ouvert.

(61) Alice a demandé des explications, et pourtant on ne lui a pas répondu.

(62) Max a argumenté, (mais + pourtant) il n'a pas convaincu.

(63) Ce train va vers Paris, (mais + pourtant) il ne va pas à Paris.

(64) Titi est un oiseau, (mais + pourtant) il ne vole pas.

(65) Max est un bon joueur de tennis, (mais + pourtant) il perd souvent.

tous cas correspondant à des topoï dont j'ai à plusieurs reprises affirmé un aspect intrinsèque d'ailleurs facilement vérifiable. Pour rendre compte de ce phénomène, je raisonnerai sur l'exemple simple (et même simpliste) :

(66) Max cherche, (mais + et pourtant) il ne trouve jamais rien.

en supposant par exemple que Max se livre à des recherches de type scientifique. Dans un tel contexte, tant mais que pourtant introduisent un conséquent $\sim \mathrm{n}=$ Max ne trowve jamais rien qui s'oppose à ce qui pourrait être tiré de l'antécédent $\mathrm{m}=$ Max cherche, à savoir Max trouve, sur la base du garant $\mathrm{g}(\mathrm{m}, \mathrm{n})=$ Qui cherche trouve. Dans les deux cas, la présence de $\sim \mathrm{n}$ est destinée à faire savoir que la combinaison de $\mathrm{g}(\mathrm{m}, \mathrm{n})$ et de $\mathrm{n}$ n'est pas suffisante pour qu'on puisse en tirer $\mathrm{n}$. Or nous avons vu qu'un tel phénomène a en fait deux interprétations : dans la première, on ne peut tirer $n$ parce que l'antécédent m n'est pas suffisamment fort, bien que soit valide le garant $\mathrm{g}(\mathrm{m}, \mathrm{n})$. En d'autres termes, Max cherche laisse passer trop de possibles exceptions à Qui cherche trouve pour que la conclusion Max trouve soit assurée. Le conséquent $\sim \mathrm{n}$ présente donc une exception ordinaire à g $(\mathrm{m}, \mathrm{n})$, et correspond par ailleurs à une lecture déréalisante. Dans la seconde interprétation, c'est le garant luimême qui est en cause. Malgré une attitude appropriée, Max ne va rien trouver parce que le sujet choisi est stérile, ou encore qu'il joue constamment de malchance. Dans ces conditions, le conséquent $\sim$ présente cette fois une exception extraordinaire, qui invalide le garant et ne correspond donc plus à une lecture déréalisante. Si nous forçons chacune des deux lectures par le biais de conclusions adéquates, on s'aperçoit alors que la première interprétation correspond typiquement à l'usage de mais, et empêche pourtant. Et que la seconde correspond cette fois à l'usage caractéristique de pourtant, bien que mais reste possible. Vérifions-le :

(57) Max est un chercheur médiocre: il cherche, (mais + ??et pourtant) il ne trouve jamais rien.

(58) Max n'a vraiment pas de chance dans ses recherches: il cherche, (mais + et pourtant) il ne trouve jamais rien. 
Mais/pourtant dans la contre-argumentation directe : raisonnement, généricité et lexique

(59) Ce train est uniquement de banlieue : il va vers Paris, (mais + ??et pourtant) il ne va pas à Paris.

(60) Attention avec ce train : il va vers Paris, (mais + et pourtant) il ne va pas à Paris.

(61) Inutile de courir pour attraper Titi : c'est un oiseau, (mais + ??et pourtant) il ne vole pas.

(62) C'est quand même un peu bizarre: Titi est un oiseau, , (mais + et pourtant) il ne vole pas.

On voit clairement sur ces exemples que la lecture déréalisante correspond à une exception ordinaire, i.e. au choix du topos intrinsèque comme cadre de l'enchaînement : il permet l'usage de mais, et empêche celui de pourtant. Dans le cas de l'exception extraordinaire, il n'y a plus lecture déréalisante, et l'usage de pourtant redevient possible. Revenons maintenant à :

(56) Les travaux de la maison avancent, (mais + ??pourtant) ils avancent lentement.

Etant donné que avancer lentement présuppose avancer, et que le présupposé joue un rôle proche de celui d'un thème, ce présupposé est cadre discursif de (56), et impose les topoï intrinsèques qui lui sont liés. D'où la possibilité de mais et l'impossibilité de pourtant.

On voit les différentes conclusions qu'on peut tirer de ce qui précède. D'une part, l'affirmation selon laquelle (et) pourtant ne peut pas se combiner avec un topos intrinsèque est erronée. Ce qu'il faut en fait dire, c'est qu'une telle combinaison est impossible dans une contre-argumentation déréalisante, qui ne supporte que mais. Elle redevient possible dès lors que la contre-argumentation a pour but la disqualification du garant. Dernière conclusion : on aura sans doute remarqué que je n'ai ici utilisé le mot topos que par commodité. Je n'ai en fait jamais utilisé la notion de topos de l'argumentation dans la langue. Tout ce qui est dit dans cette étude reste parfaitement valide si on remplace topos par phrase générique appartenant au stéréotype d'un terme, au sens que j'ai donné plus haut à ces notions. 
Jean-Claude Anscombre

\section{BIBLIOGRAPHIE}

ANSCOMBRE, Jean-Claude, (1983) «Pour autant, pourtant (et comment) : à petites causes, grands effets », Cahiers de linguistique française, Genève, $\mathrm{n}^{\circ}$ 5, pp.37-85.

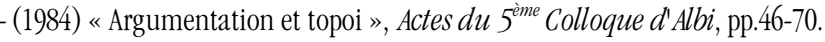

- (1989) «Théorie de l'argumentation, topoï, et structuration discursive », Revue québécoise de linguistique, $18, \mathrm{n}^{\circ}$ l, pp.13-56.

- (1990) « Les syllogismes en langue naturelle : déduction logique ou inférence discursive? », Cahiers de linguistique française, Genève, $n^{\circ} 11, \mathrm{pp} .215-240$.

- (1991) «Dynamique du sens et scalarité », in: L'argumentation, Collection «Philosophie et langage », A.Lempereur éd., Ed. Mardaga, Liège-Paris, pp.123-146.

- (1993) « Temps linguistique et théorie des topoï », in Lieux communs, topoï, stéréotypes, clichés, C.Plantin éd., Ed. Kimé, Paris, pp.271-289.

- (1995a) « La théorie des topoï : sémantique ou rhétorique? », Hermès, n 15, pp.185-98.

- (1995b) « Topique or not topique : formes topiques intrinsèques et formes topiques extrinsèques », Journal of Pragmatics, 24, pp.115-141.

- (1995c) La théorie des to poï, J.C.Anscombre éd., Ed. Kimé, Paris.

- (1996a) «Semántica y léxico: topoi, estereotipos y frases genéricas», Revista española de lingü̈stica, 25, n², pp.297-310.

- (1996b) «Développements récents de la théorie des topoï », in L'Argumentation dans avec ou malgré la langue. Actes de la journée d'études coordonnée par D. Desmarschelier à l'Université René Descartes (13 mai 1995), pp.37-56.

- (1998) «Pero/sin embargo en la contra-argumentación directa : razonamiento, genericidad y léxico », Signo y Seña, nº, pp.75-104.

- (2001a) « Le rôle du lexique dans la théorie des stéréotypes », Langages, n¹42, pp.57-76.

- (2001b) «La nuit, certains chats sont gris, ou la généricité sans syntagme générique », dans Hommage à M.Galmiche, à paraître dans Linx.

- (2001c) « Dénomination, sens et référence dans une théorie des stéréotypes nominaux », Cahiers de praxématique, n³6, pp.43-72.

ANSCOMBRE, Jean-Claude ; DUCROT, Oswald, (1977) « Deux mais en français », Lingua, 43, pp.23-40.

- (1978) « Lois logiques et lois argumentatives I », Français Moderne, 46, n4, pp.347-57.

- (1979) « Lois logiques et lois argumentatives II », Français Moderne, 47, n¹, pp.35-42.

- (1983) L'argumentation dans la langue, Ed. Mardaga, Bruxelles-Liège-Paris.

- (1986) « Argumentativité et informativité », in De la métaphysique à la rhétorique : in memoriam Ch.Perelman, M.Meyer éd., Ed. de l'Université Libre de Bruxelles, pp.79-94. 
Mais/pourtant dans la contre-argumentation directe : raisonnement, généricité et lexique

DUCROT, Oswald, (1988) «topoï et formes topiques », Bulletin d'études de linguistique française, Tokyo, $\mathrm{n}^{\circ} 22$, p. $1-14$.

- (1995) « Les modificateurs déréalisants », Journal of Pragmatics, 24, pp.145-65.

FRADIN, Bernard, (1984) « Anaphorisation et stéréotypes nominaux », Lingua, n ${ }^{\circ}$ 64, pp. 325-369.

GALMICHE, Michel, (1983) « Les ambiguités référentielles ou les pièges de la référence », Langue française, 57, pp. 60-86.

- (1985) « Phrases, syntagmes et articles génériques », Langages, nº 85, pp. 2-39.

GARCÍA NEGRONI, Marta, (1995) « Scalarité et réinterprétation : les modificateurs surréalisants », in La théorie des topoi, J.C.Anscombre éd., Paris, Ed. Kimé, pp.101-144.

KLEIBER, Georges, (1988) «Phrases génériques et raisonnement par défaut », Français Moderne, 56, n¹/2, pp. 1-15.

- (1989) «Généricité et typicalité », Français Moderne, 57, n 3/4, pp. 127-154.

PUTNAM, Hilary, (1975) « The Meaning of 'Meaning' », Philosophical Papers, vol.2, Cambridge University Press, pp. 215-271.

-(1990) Représentation et réalité, Coll. Nrf. Essais, Ed. Gallimard, Paris.

PORTOLÉS, José, (1995) «Diferencias gramaticales y pragmáticas entre los conectores dicursivos pero, sin embargo y no obstante ", Boletín de la Real Academia Española, Tomo LXXV, Cuaderno CCLXV, pp. 231-269.

ROULET, Eddy, et alii, (1985) L'articulation du discours en français contemporain, Berne, Ed. Peter Lang. 
